\title{
An Algorithm for the Spatiotemporal Dynamic of Insect Pest in Complex Agricultural System
}

\author{
Ritter A. Guimapi \\ Department of computing, School of Computing and \\ Information Technology \\ Jomo Kenyatta University of Agriculture and \\ Technology (JKUAT) \\ Nairobi, Kenya
}

\author{
George O. Okeyo \\ Department of computing, School of Computing and \\ Information Technology \\ Jomo Kenyatta University of Agriculture and \\ Technology (JKUAT) \\ Nairobi, Kenya
}

\begin{abstract}
Nowadays, our living environment is becoming more and more complex and, the complexity can be found in almost all the aspects of our daily activities Agroecosystems are good examples of complex systems, and the control of insect invasion is essential in pest management activities. Species distribution modeling(SDM) is a widely used modelling approach that predict the distribution of insect pest across geographical location. Despite their ability to predict the different zone suitable for the spatial dispersion of insects, there are unable to tell us about the time the invasion may occur in these location. this is why a new spatiotemporal algorithm is proposed to improve prediction of insect invasion. Afterward the algorithm is implemented and some simulation case of its application are presented.
\end{abstract}

Keywords: agroecosystem, Spatiotemporal process, Insect pest dispersal, optimization algorithm, prediction

\section{INTRODUCTION}

Nowadays, our living environment is becoming more and more complex and, the complexity can be found in almost all the aspects of our daily activities; this include sport [1-3] economy and finance[4-6], politics[7], health, society[8], agriculture[9-11] etc. There is no formal definition about what a system is, but it can be defined as a collection of interdependent elements organized for the achievement of a goal. Many real world systems, including living microorganisms, human society and many others are better understood when looked at as complex system where useful knowledge derive from the interaction among components of the system [12]. Complex systems increase the complexity and the uncertainty of understanding and interpretation for managers and policy maker when analyzing underlining processes to make decisions.

Understanding underlining processes in complex systems is of paramount importance as it will help for early detection of wrong behaviors of the system and understand why it does not behave as expected. Mathematical and computational modeling has become the keys approach to represent these systems using models and simulations [13-16]. However, the skills and knowledge required for designing useful mathematical model are not common into many researches team especially those related to fields of medical, biological or agricultural sciences. Therefore, the best approach consists to embed these processes into a Decision Support System(DSS)[15,17] to assist policies makers simulate and analyze real world problems[18] DSS is defined as interactive computer-based systems that help decision makers utilize data and model to solve unstructured problems[19,20]; Sprague and Carlson, 1982). It became a complete area of research in the middle of 1970[21] and has since gradually gained a lot of attention among scientific community.

Agroecosystems are good examples of complex systems and, it represents a cropping expanses well adapted to a particular geographic region[22]. The definition usually depend on the context and the purpose but put emphasis on elements that include the geographic area, weather pattern, natural resources, cropping pattern and products [22]. The development and maintenance of a good agroecosystem requires to preserve complex interactions and dynamics among its keys components. This include: water, terrain and soils, climate, nutrients and also living organisms(Crops, human, animals), [22]. The complexity of these interaction make agroecosystems one of the most difficult complex system to analyze and understand for all the stakeholders involved. Indeed, insects are central to the performance of many agroecosystem processes. The structure and social organization of insect colonies have been explored in many way as a complex system[9-11]. However, it is in their role as herbivores that conflicts arise with agricultural production due to direct consumption of cultivated crops and indirect damage by plant virus transmission or spoilage of potential yield[23].. To avoid or reduce damage on crops, farmers apply chemical pesticides against pests. Despite its ability to quickly suppress pest, the use of chemical pesticide presents too many disadvantages as it pollutes air water and soil; it contributes to the development of resistance among the target pest[24] people applying these pesticides are sometime exposed to dangerous concentration of chemical which affect their health [25].

Having a good production require the implementation of efficient pest management strategies. The farmers, who play the key role in the system need to quickly adjust themselves to handle farming activities which are becoming more and more complex. Being an excellent crop producer also require the abilities to embed some agro ecological concepts and principles for the management of agricultural systems. This implies optimizing the interactions between plants, animals, humans and the environment while considering the social aspect that need to be addressed for a sustainable food system. Hence, natural biodiversity of agroecosystem must be studied as a complex system with the goal to understand, predict and take advantage of the self-emerging behavior from various interactions of the components of the system. This indeed, is not an easy task and therefore require the collaboration among 
farmers, researchers and extension officers to develop tools to easy or improve decisions making process.

\section{PROBLEM STATEMENT}

Pest management activities/practice are essential for the survival of agroecosystems, and for the success of agricultural production; therefore, require rapid adoption of modern strategies and approaches to monitor insect invasion. The attempt to protect plant from insect's pest is centered too much on the insect instead of looking at all the interacting component of the agricultural system.

Species distribution modeling(SDM) is a widely used modelling approach that predict the distribution of insect pest across geographical location using climate data such temperature, relative humidity, precipitation. These climate data are combined with physiological information of the species of concern, generally obtained from location where the pest was collected while successfully developing and reproduce. Maxent and Climex are the two famous software serving as tool to design SDM. Despite their ability to predict the different zone suitable for the spatial dispersion of insects, there are unable to tell us about the time the invasion may occur in these location. Therefore, the challenge being addressed here consists to propose a new algorithm to predict the spatiotemporal dispersal of a given pest. This will improve the previous one by not providing only the spatial prediction about where the pest can invade, but will also provide a temporal prediction about when that invasion will occur.

\section{ALGORITHM DESCRIPTION}

The proposed algorithm is based on cellular automat(CA). The reason of this choice is because the states of the CA will allow to enumerate the different states of the process of insect invasion. The rules of the CA will ease the design of the mechanism for insect dynamic of dispersal. Moreover, the properties of the neighborhood offer by the CA allow to adjust the flight ability of the targeted insect for a better prediction of the invasion. Let Susceptible(S), Exposed(E) and Invaded (I) the three possible state of a given location during the process of insect invasion. Susceptible locations are area where the insect has never crossed by or settled to reproduce and cause damage on crops. Exposed is the state of locations by where the best transit to reach a location with suitable environmental conditions; and finally Invaded location are those where environmental condition are affordable, therefore, the pest settle and cause damage on agricultural crops. The main environmental factor considered here are temperature, relative humidity, vegetation, presence of the host plant.

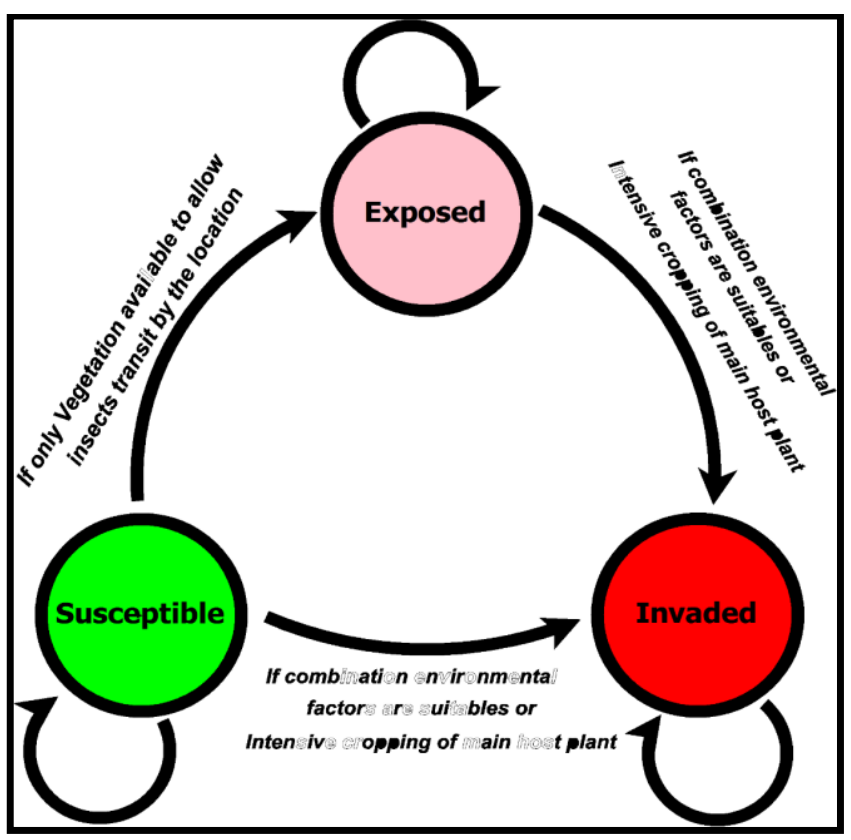

Figure 1: State transition diagram for insect dispersal

Based on these different states and the environmental factors, the new algorithm was proposed and presented as follows: 


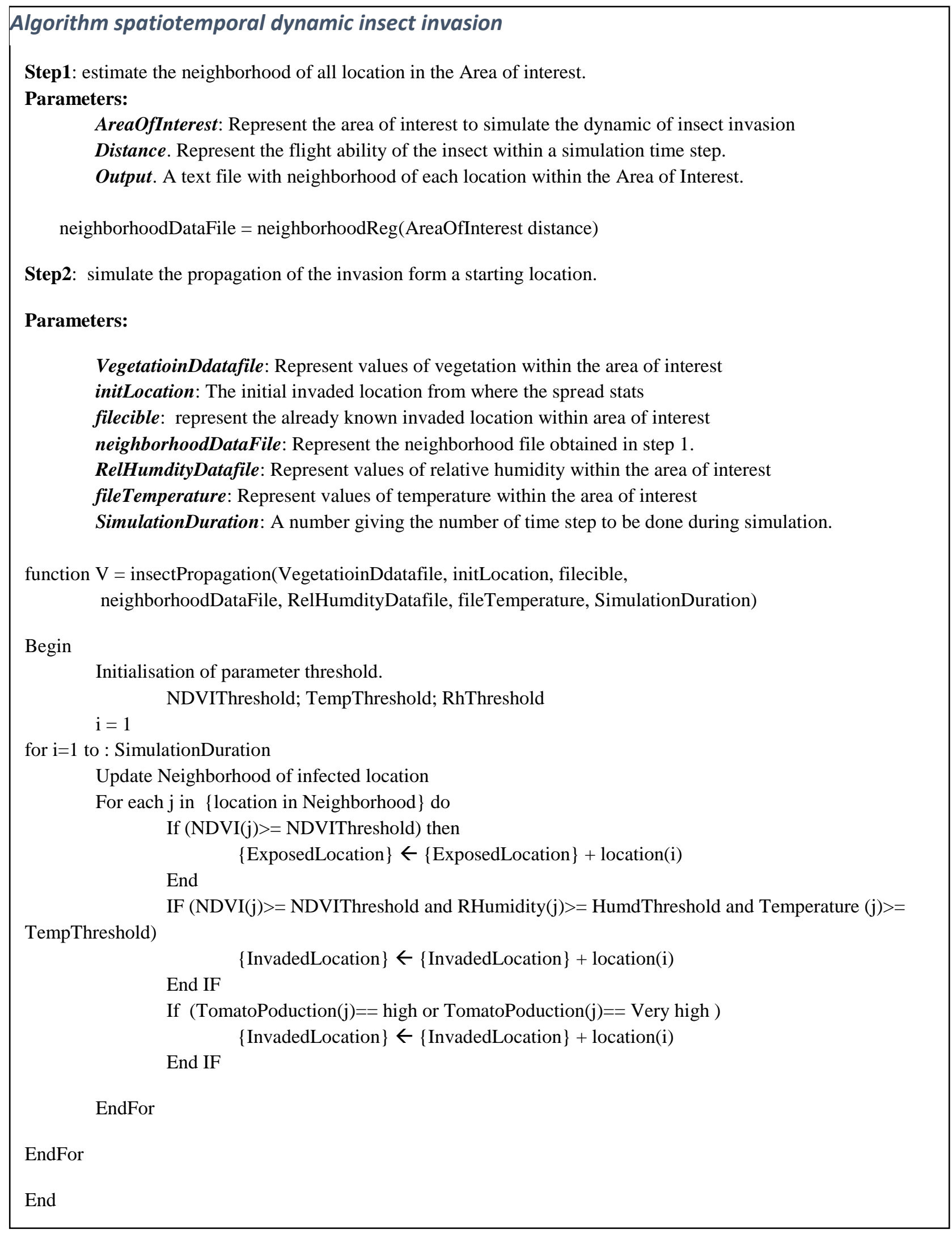




\subsection{Algorithm implementation and simulation}

MATLAB (R2010a, The Math-Works) was used as programming language to implement the algorithm and proceed with simulations. Mapping Toolbox ${ }^{\mathrm{TM}}$ implement a lot of algorithm useful for handling and analyzing geospatial data. Once the implementation of the algorithm was done, the function was used to simulate the invasion of an invasive insect pest named Tuta absoluta (Meyrick 1917) both in Africa and Asia. The algorithm successfully predicted that the pest will invade the southern part of Africa in 2017 and was later confirmed by Visser et al. (2017) in one of their resent work.[26]. Fig.2 bellow summarize the result of simulation obtained using our algorithm.

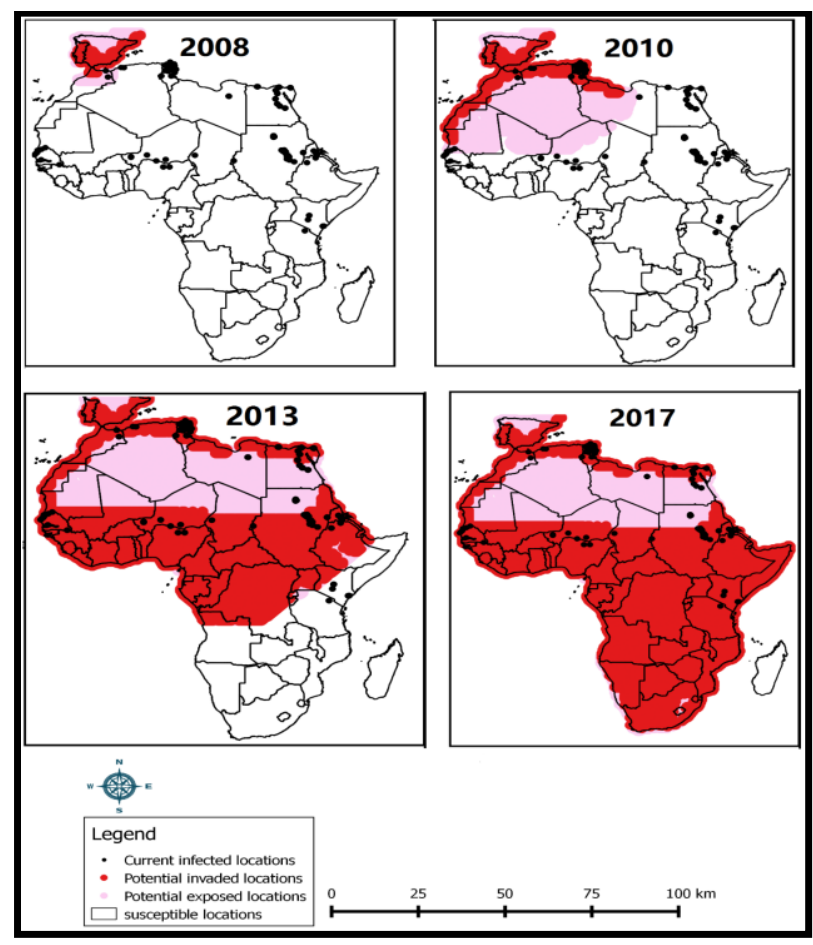

Figure 2: Simulation of the algorithm to predict tuta absoluta invasion in Africa

\section{DISCUSSION AND CONCLUSION}

The protection of agricultural crops against insect pests require new approaches that will ease the prediction in time when the invasion may reach a given location. Most of the currently existing techniques focus more on estimating suitable location for the development and the survival of the pest based on the presence data recorded of the pest.

The algorithm presented here has the advantage to be able to predict also the suitable locations for insect survival. To do that it only requires as input parameter to select those that favor the establishment of the pest and therefore, proceed with simulation into the area of concern. Once of the key benefice of the algorithm is the flexibility it offers for the input parameter which can help during simulation to analyze the effect of one climate factor on another one. The implementation was done to allow the user during simulation to cancel or the effect of one input parameter.

After showing satisfactory result for predicting the dynamic of invasion in Africa, it would be great to explore how the algorithm perform on other continent such Asia for example where the pest is still in progress. Moreover, given that the application was done in this case on a very large scale space and on a pest with very high ability of flight, some text also has to be done on small scale land. The main challenge here will definitely be the availability of relate data.

Despite providing satisfactory result, this algorithm can still be improved. The rules of the CA can be updated in a way such it can take into account the phenology of the targeted insect. This main advantage doing that will be the improvement of the efficacy in prediction the time of invasion from one location to another. Moreover, other platform dealing with the prediction of insect invasion should consider the inclusion of the approach presented in their platforms. 
[13] N. Boccara, Modeling complex systems, Springer, 2010.

https://books.google.co.ke/books?id=ML2RNAEAC AAJ\&source=gbs_book_other_versions (accessed January 10, 2018).

[14] Z. Ji, K. Yan, W. Li, H. Hu, X. Zhu, Mathematical and Computational Modeling in Complex Biological Systems, Biomed Res. Int. 2017 (2017) 1-16. doi:10.1155/2017/5958321.

\section{REFERENCES}

[1] N. Balague, C. Torrents, R. Hristovski, K. Davids, D. Araújo, Overview of complex systems in sport, J. Syst. Sci. Complex. $26 \quad$ (2013) 4-13. doi:10.1007/s11424-013-2285-0.

[15] R.M. Peart, R.B. Curry, Agricultural systems modeling and simulation, Marcel Dekker, 1998 https://books.google.co.ke/books/about/Agricultural_ Systems_Modeling_and_Simula.html?id=gQL1a6y4 W9YC\&redir_esc=y (accessed December 20, 2017). effective teams, Int. J. Comput. Sci. Sport. 2 (2003) $8-15$.

http://necsi.edu/projects/yaneer/SportsBarYam.pdf (accessed January 10, 2018).

[3] K. (Keith) Davids, Complex systems in sport., 2014.

[4] J.D. Farmer, Economics needs to treat the economy as a complex system, (2012). https://www.colorado.edu/AmStudies/lewis/ecology/ needeconcomplex.pdf (accessed January 10, 2018).

[5] J. Foster, From Simplistic to Complex Systems in Economics, (2004). http://www2.econ.iastate.edu/tesfatsi/MacroAsNetwo rk.Foster.pdf (accessed January 10, 2018).

[16] H. Sayama, Introduction to the Modeling and Analysis of Complex Systems, (2015) 477. doi:10.1017/CBO9781107415324.004.

[17] D.J. Power, Building Model-Driven Decision Support Systems, J. Power. (2000) http://dssresources.com/subscriber/password/ch9mds s.pdf (accessed January 10, 2018).

[18] Mago, Computational Models of Complex Systems, Springer International Publishing, Cham, 2014. doi:10.1007/978-3-319-01285-8.

[19] T. Raksha, Decision \{Support $\}$ \{System $\}$ (\{DSS $\}$ ): \{Concept\}, \{Characteristics\} and \{Benefits\}, YourArticleLibrary.Com Next Gener. Libr. (2015). http://www.yourarticlelibrary.com/business/decisionsupport-system/decision-support-system-dssconcept-characteristics-and-benefits/69757/ (accessed April 4, 2017).

[7] Y.P. Rhee, Complex systems approach to the study of politics, Syst. Res. Behav. Sci. 17 (2000) 487-491. doi:10.1002/1099-1743(200011/12)17:6<487::AIDSRES350>3.0.CO;2-E.

[8] N. Bellomo, G. Ajmone Marsan, A. Tosin, Complex Systems and Society, Springer New York, New York, NY, 2013. doi:10.1007/978-1-4614-7242-1.

[9] S.I. Caramihai, I. Dumitrache, Agricultural enterprise as a complex system: A cyber physical systems approach, in: Proc. - 2015 20th Int. Conf. Control Syst. Comput. Sci. CSCS 2015, IEEE, 2015: pp. 659-664. doi:10.1109/CSCS.2015.147.

[10] H. van Mil, E. Foegeding, Using a complex system approach to address world challenges in Food and Agriculture, ArXiv Prepr. ArXiv .... 9 (2013) 1-13. https://arxiv.org/ftp/arxiv/papers/1309/1309.0614.pdf (accessed January 10, 2018).

[11] R. Taylor, Agriculture is complex. That's what makes it adaptive | Down to Earth on WordPress.com, (2014). https://rachaelctaylor.wordpress.com/2014/10/14/agri culture-is-complex-thats-what-makes-it-adaptive/ (accessed January 10, 2018).

[12] J. Ladyman, J. Lambert, K. Wiesner, What is a complex system?, 2013. doi:10.1007/s13194-0120056-8.

[20] R.H. Sprague, E. Carlson, Building \{Effective \{Decision\} \{Support\} \{Systems\}, Prentice Hall College Div, Englewood Cliffs, N.J, 1982.

[21] A. Gachet, Building \{Model $\}$ \{Driven $\}\{$ Decision $\}$ \{Support\} \{Systems\} with \{Dicodess\}, vdf Hochschulverlag AG, 2004.

[22] K.R. (Kowligi R.. Krishna, Agroecosystems : soils, climate, crops, nutrient dynamics, and productivity, 2013.

https://www.google.com/search?q=Krishna+...Agroe cosystems $\% 3 \mathrm{~A}+$ Soils....+and+Productivity\&ie=utf$8 \&$ oe $=u t f-8 \&$ client $=$ firefox $-b \quad$ (accessed November $29,2017)$

[23] M. Shah, Z. Chitforoushzadeh, K.A. Janes, Statistical Data Analysis and Modeling, in: 2016: pp. 155-175. doi:10.1007/978-3-319-21296-8_6.

[24] G.P. Georghiou, Management of Resistance in Arthropods, in: Pest Resist. to Pestic., Springer US, Boston, MA, 1983: pp. 769-792. doi:10.1007/978-14684-4466-7_32.

[25] M.W. Aktar, D. Sengupta, A. Chowdhury, Impact of pesticides use in agriculture: their benefits and hazards., Interdiscip. Toxicol. 2 (2009) 1-12. doi:10.2478/v10102-009-0001-7. 
International Journal of Computer Applications Technology and Research Volume 8-Issue 02, 52-57, 2019, ISSN:-2319-8656

[26] D. Visser, V. Uys, R. Nieuwenhuis, W. Pieterse, First records of the tomato leaf miner Tuta absoluta (Meyrick, 1917) (Lepidoptera: Gelechiidae) in South Africa, BioInvasions Rec. 6 (2017) 301-305. doi:10.3391/bir.2017.6.4.01. 